\title{
BMJ Open Rehabilitation and reintegration programming adjunct to female genital fistula surgery: a scoping review protocol
}

\author{
Alison M El Ayadi (10 , ${ }^{1}$ Caitlyn E Painter, ${ }^{1,2}$ Alexandre Delamou, ${ }^{3}$ Jill Barr-Walker, ${ }^{4}$ \\ Susan Obore, ${ }^{5}$ Josaphat Byamugisha, ${ }^{5,6}$ Abner Korn, ${ }^{1}$ Justus K Barageine ${ }^{5,6,7}$
}

To cite: El Ayadi AM, Painter CE, Delamou A, et al. Rehabilitation and reintegration programming adjunct to female genital fistula surgery: a scoping review protocol. BMJ Open 2019;9:e027991. doi:10.1136/ bmjopen-2018-027991

- Prepublication history and additional material for this paper are available online. To view these files, please visit the journal online (http://dx.doi. org/10.1136/bmjopen-2018027991).

7th International Society of Obstetric Fistula Surgeons Conference, December 5-9, 2018: Kathmandu, Nepal

Received 16 November 2018

Revised 22 July 2019

Accepted 25 September 2019

Check for updates

(C) Author(s) (or their employer(s)) 2019. Re-use permitted under CC BY-NC. No commercial re-use. See rights and permissions. Published by BMJ.

For numbered affiliations see end of article.

Correspondence to

Dr Alison M El Ayadi; alison.elayadi@ucsf.edu

\section{ABSTRACT}

Introduction Female genital fistula is a debilitating traumatic injury, largely birth-associated, globally affecting up to 2 million women, mostly in sub-Saharan Africa. Fistula has significant physical, psychological and economic consequences. Women often face challenges in reintegrating and resuming prior roles despite successful surgery. Synthesising the evidence on services adjunct to fistula surgery and their outcomes is important for developing the evidence base for best practices and identifying research priorities. This scoping review seeks to examine the range of rehabilitation and reintegration services provided as adjunct to genital fistula surgery, map the existing programming and outcomes, and identify areas for additional research. Methods and analysis Our scoping review is informed by existing methodological frameworks and will be conducted in accordance with Preferred Reporting Items for Systematic Reviews and Meta-Analyses-ScR guidelines. The search strategy will be applied to nine biomedical, public health and social science databases. The initial search was completed on 27 September 2018. Grey literature will be identified through targeted Google searches and from organisational websites identified as relevant by the United Nations Population Fund (UNFPA) Campaign to End Fistula. We will iteratively build our search strategy through term harvesting and review, and search reference lists of reports and articles to identify additional studies. Two reviewers will independently screen titles and abstracts, followed by full-text screening of all potentially relevant articles and standardised data extraction. Articles eligible for inclusion will discuss research or programmatic efforts around service provision in adjunct to surgery among females with genital fistula. Data will be presented in summary tables accompanied by narrative description. Ethics and dissemination Ethics approval is not required for a scoping review. Our results can be used to inform policy, serve as support for funding and development of reintegration programmes and highlight areas for subsequent research. Results will be disseminated at relevant conferences and published in a peer-reviewed journal.

\section{INTRODUCTION}

Female genital fistula is a debilitating and traumatic injury that affects an estimated
Strengths and limitations of this study

- No systematic efforts have been previously carried out to map the rehabilitation and reintegration services provided in adjunct to female genital fistula surgery despite recognition in the literature of significant physical, psychosocial and economic sequelae of the condition.

- Our scoping review uses an established framework, employs a comprehensive search strategy developed iteratively in conjunction with a medical librarian, and study selection and extraction will be undertaken by content experts.

- Compiling the evidence on existing programmes and outcomes will inform the development of evidence-based public health strategies for supporting women recovering from female genital fistula and inform the measurement of impact of such strategies.

- No formal assessment of the quality of studies will be conducted, as the goal of a scoping review is to map the range of evidence on a particular topic.

- The findings of this scoping review will be limited to articles and programme reports published in English or French.

2 million women, mostly in sub-Saharan Africa. ${ }^{1}$ Primarily due to prolonged and neglected obstructed labour from cephalopelvic disproportion or malpresentation combined with delays in accessing comprehensive emergency obstetric care, up to 100000 new cases occur each year globally. During the obstructed labour, ischaemia from compression of soft vaginal, bladder or rectal tissue between the fetal head and pelvic bone results in ischaemic necrosis, and a fistula is formed on sloughing of this tissue. ${ }^{2}$ Women with fistula experience uncontrollable leakage of urine and/or faeces, which contributes to the development of genital sores and infection. ${ }^{34}$ In addition to pain and general weakness, ${ }^{5}$ women may experience nerve damage, uterine cervix injuries and 
pelvic bone trauma which present as secondary infertility and gait disorders. ${ }^{6}$ For most deliveries resulting in fistula, the baby does not survive. ${ }^{7}$ Women with fistula are often stigmatised and marginalised from their families and communities and live in isolation, unable to participate in social, economic, food preparation or religious activities, ${ }^{57}$ and report high psychiatric morbidity including depression, which may persist after surgery. ${ }^{8-10}$

Access to obstetric fistula surgery has improved in sub-Saharan Africa overall; however, despite having undergone surgical repair, women may continue to face myriad physical and psychological challenges to resuming their previous roles or adjusting to new circumstances. They may also require further medical care depending on the severity of their injuries and surgical outcomes and require healthcare access for subsequent pregnancies and births. Longitudinal studies from sub-Saharan Africa have identified risks of adverse outcome following fistula surgery, including fistula recurrence, persistent fistula-related symptoms, subsequent fertility challenges and adverse perinatal outcomes. ${ }^{11-13}$ In Guinea, for example, the cumulative incidence of fistula recurrence was $16 \%$ at 24 months $^{13}$. In Uganda, by 12 months postsurgery, one-third of women continued to experience urinary incontinence, $17 \%$ reported weakness and $9 \%$ reported general pain. ${ }^{14}$ In Malawi, only one-fifth of women with reproductive potential were able to become pregnant in the year following surgery. ${ }^{12}$ Furthermore, experience of persistent physical symptoms or adversity is associated with substantially lower psychosocial health. ${ }^{14}$ All of these factors limit women's ability to resume their previous roles despite successful surgery, particularly in the context of economic hardship. ${ }^{15}$ A systematic review of 10 qualitative studies from sub-Saharan Africa identified various reintegration needs from the perspectives of both women affected by fistula and health professionals working with this patient population, including health education and counselling. ${ }^{16}$

This literature highlights the need for postsurgical reintegration and rehabilitation services; however, there remains a knowledge and practice gap around programming to best assist women to reintegrate into their families and communities after surgery. Preliminary evidence from Nigeria and Eritrea supports short-term facility-based psychological intervention for improving women's mental health, ${ }^{17} 18$ and programming supported by non-governmental organisations often includes counselling, physical therapy and skills training. Synthesising research on the reintegration process, evaluation and service provision is of paramount importance to develop a strong evidence base, which can guide prioritisation of service provision to meeting the future health needs of women who have experienced obstetric fistula.

Thus, the objective of this scoping review is to examine the range of rehabilitation and reintegration services provided as adjunct to genital fistula surgery, map the existing programming and outcomes, and identify areas where additional research is necessary.

\section{METHODS AND ANALYSIS}

The purpose of this scoping review is to understand the extent to which rehabilitation and reintegration services have been provided to women in conjunction with female genital fistula surgery and the outcomes of such programming. The findings from this review will be used to support the evidence-based development of programming for women undergoing surgery for female genital fistula surgery and identify further research needs in this area. A preliminary search for existing reviews on this topic has been conducted in Joanna Briggs Institute Database of Systematic Reviews and Implementation Reports (JBISIR), PROSPERO, PubMed, Cochrane Database of Systematic Reviews, EPPI and Epistemonikos, and no similar systematic or scoping reviews were identified. Given the lack of prior reviews, a scoping review is an appropriate first step to assessing the literature in this area. Our scoping review methodology is informed by the Arskey and $\mathrm{O}^{\prime}$ Malley ${ }^{19}$ and Levac et $a l^{20}$ frameworks and will be conducted in accordance with Preferred Reporting Items for Systematic Reviews and Meta-Analyses (PRISMA-ScR) guidelines. ${ }^{21}$

\section{Patient and public involvement}

Patients and/or public were not involved in the design or development of this protocol and research questions. Results of this study will not be disseminated to study participants.

\section{Identifying the research question}

The objective of this scoping review is to examine the range of rehabilitation and reintegration services provided as adjunct to genital fistula surgery, map the existing programming and outcomes of programming, and identify areas where additional research is necessary. To achieve these objectives, our review targets the following research questions:

1. What rehabilitation and reintegration services are provided as adjunct to genital fistula surgery for women with obstetric, iatrogenic or traumatic fistula?

2. What are the components of each rehabilitation and reintegration intervention, and how are they delivered?

3. What is the impact of each rehabilitation and reintegration intervention on women's physical, psychosocial and economic status?

4. What are the authors' recommendations for rehabilitation and reintegration interventions and intervention delivery?

5. What are the areas of research gaps and what topics should future research focus on?

In this review, we include genital fistula from obstetric, iatrogenic and traumatic aetiologies. Obstetric refers to fistula resulting from pressure necrosis due to prolonged obstructed labour; iatrogenic refers to fistula resulting from surgical error, primarily occurring during caesarean section or hysterectomy and traumatic refers to those fistula resulting from sexual assault. 


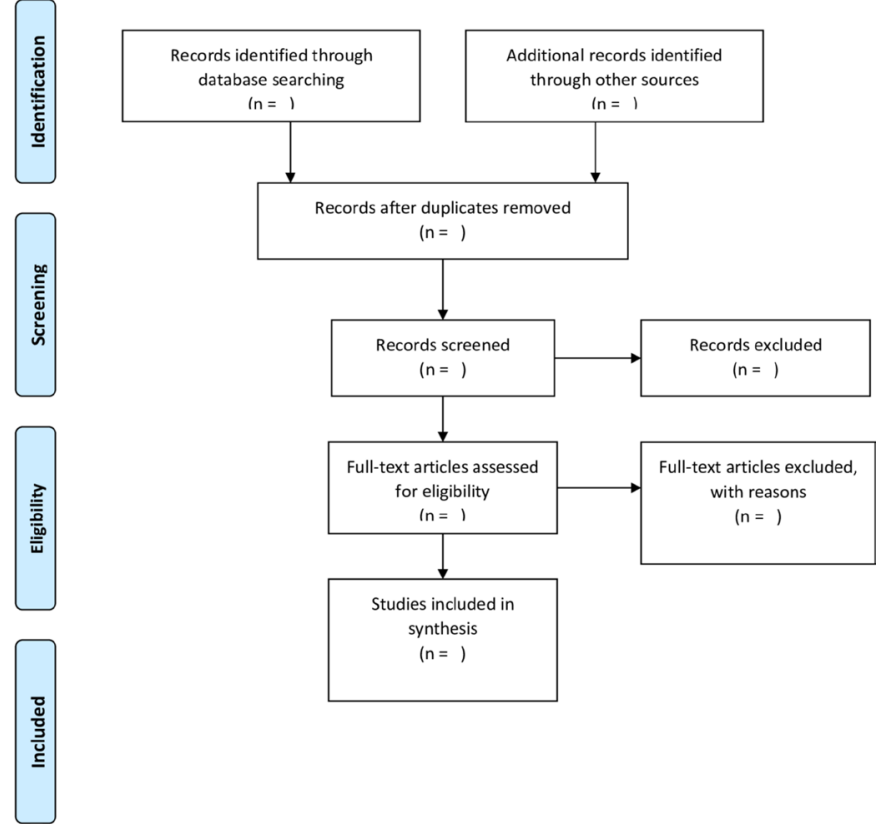

Figure 1 Preferred Reporting Items for Systematic Reviews and Meta-Analyses flowchart for selection of eligible studies and programme reports.

\section{Types of sources}

Sources to be reviewed include biomedical, public health and social science databases, including PubMed, Embase, Popline, PsycINFO, Web of Science, Sociological Abstracts, Social Services Abstracts and African Journals Online, the grey literature identified within targeted Google searches and directly from organisation websites identified as being relevant by the Campaign to End Fistula Partners (UNFPA) (online supplementary appendix 1), and through personal queries with clinical and social service organisations working in fistula research. All data and articles will be managed within Endnote citation software.

\section{Search strategy}

We will employ a three-step search strategy for identifying published and unpublished studies for our scoping review. An initial search was performed on 27 September 2018.

We will begin by conducting a preliminary search of PubMed to identify key articles on our topic and begin the process of term harvesting, described herein. From these key articles, we will extract text words and $\mathrm{MeSH}$ terms from titles, abstracts and author-supplied keywords to build a comprehensive list of keywords and controlled vocabulary terms, to inform our search strategy development. Next, we will work collaboratively with a medical librarian (JB-W) with training and experience in systematic reviews to design our search strategy using an iterative process that follows an established search method. ${ }^{22}$ Each potential search term will be tested, with the two primary reviewers examining the first 60 unique results for each term in order to determine that term's relevance and subsequent inclusion in the search strategy. The search strategy will be developed in PubMed and adapted to other databases accordingly, using controlled vocabulary (eg, MeSH, Emtree, thesaurus terms) where databases allow. A second librarian will peer review the final search strategy using the Peer Review of Electronic Search Strategies guidelines. ${ }^{23}$

Third, the reference lists of all included reports and articles will be searched to identify any additional studies. The reviewers will contact authors of primary studies, reviews and grey literature if further information is desired.

The search strategy will combine two main concepts: obstetric fistula and social reintegration. Boolean logic will be applied by combining similar key words and controlled vocabulary (eg, MeSH and Emtree terms) with OR and using AND between the two concepts, for example ("obstetric fistula" OR "vesicovaginal fistula") AND (reintegration OR rehabilitation). During preliminary searching, we have found a large number of irrelevant articles related to cancer in two sets of database (31\% of our results in Embase and $17 \%$ of our results in Web of Science); therefore, an additional concept using cancer-related terms will be added using the NOT term that will exclude these terms from our searches in those two databases. To ensure that this concept exclusion will not eliminate any relevant results, the primary reviewers evaluated the first 100 unique results that would be left out of searches that used this NOT concept. No relevant articles were discovered using this method; thus, we feel confident that this technique will only exclude irrelevant articles. The complete search strategy for all databases can be found in online supplementary appendix 2 .

In accordance with PRISMA guidelines, no language limits will be used in the search. Date limits will be applied to identify relevant studies as social reintegration for obstetric fistula is a reasonably new area of study. To determine which date limits to use, primary reviewers tested searches from various publication date ranges, looking through the first 50 unique results of each time span to judge relevance of the studies included in these dates. Using this method, we chose to include articles from 2000 through 2018.

\section{Study selection}

Two primary reviewers (AE and CP) will independently screen titles and abstracts of all articles, followed by fulltext screening and data extraction. A third reviewer (AD) will be available to resolve discrepancies and will ultimately determine if the study meets inclusion criteria for data extraction. Study screening progress will be documented in a modified PRISMA flow chart (figure 1). In accordance with established scoping review frameworks, ${ }^{19} 20$ critical appraisal of study quality will not be performed owing to the heterogeneity of results and the multi-tiered aspect of the research question.

\section{Inclusion and exclusion criteria}

Inclusion and exclusion criteria for selection of eligible studies were developed using the population, concept, 
Table 1 Population, concept and context for identification of eligible studies

\begin{tabular}{ll}
\hline Criteria & Description \\
\hline Population & $\begin{array}{l}\text { Females undergoing surgery for genital fistula. } \\
\text { Fistula aetiologies: obstetric, iatrogenic (non- } \\
\text { cancer), traumatic. } \\
\text { All ages. }\end{array}$ \\
& $\begin{array}{l}\text { Any research or service provision in addition to } \\
\text { surgery; no limitations on intervention type. } \\
\text { Clinical or patient-reported outcomes beyond } \\
\text { surgical success. }\end{array}$ \\
& $\begin{array}{l}\text { Studies or reports including original research or } \\
\text { programme data. }\end{array}$ \\
Context & All contexts; articles written in English or French. \\
\hline
\end{tabular}

context elements proposed for scoping reviews by the Joanna Briggs Institute (table 1). ${ }^{24}$ We selected this approach in order to broadly identify all data that are potentially relevant to our objective.

Types of participants

Females diagnosed with genital fistula with obstetric, iatrogenic (non-cancer) or traumatic aetiology and accessing surgical treatment. No limitations on geography or age will be placed.

\section{Concept}

Research or service provision for all services provided in adjunct to surgery among females diagnosed with genital fistula of obstetric, iatrogenic (non-cancer) or traumatic aetiology. Interventions of interest are anticipated to include but will not be limited to the following: psychological therapy, physical therapy, skills training or other economic empowerment.
The scoping review will be heavily focused on the services provided; however, we will capture any clinical or patient-reported outcomes beyond surgical success including but not limited to: persistent incontinence, pain, weakness, difficulty walking, quality of life, depressive symptoms, self-esteem, relationship stability and stigma.

\section{Context}

No contextual limitations will be placed on the scoping review; however, we are unable to review articles written in languages other than English and French. Due to the geographic distribution of female genital fistula, we anticipate that the majority of the evidence will originate in sub-Saharan Africa.

\section{Extraction of results}

We have developed a draft charting table (table 2) to record key information from each publication or report. The extraction form will be pilot tested by each of the two primary reviewers with several studies to ensure that all relevant data are extracted and will be updated if additional relevant variables are identified.

\section{Data synthesis}

We will present the search and screening flow process (figure 1). The findings from the scoping review will be presented in a table summarising the information of each of the included studies to highlight year of the literature, countries of origin, intervention component and research methods, following the general format of our charting table (table 2). Additionally, we will present participant outcomes by type of intervention component and by fistula aetiology, if possible. Narrative summaries of each of these findings will accompany the figures/tables. Two reviewers (AE and $\mathrm{CP}$ ) will thematically analyse the

Table 2 Data charting domains and descriptions to be used for data extraction

\begin{tabular}{ll}
\hline Domain/subdomain & Description \\
\hline Article details & \\
\hline Article citation & Citation details of studies or report. \\
\hline Article type & Study type including empirical research studies, case studies or programme reports. \\
\hline Country & Country the article is from. \\
\hline Study details & \\
\hline Study design & Study design of empirical research articles or evaluation design for programme \\
& reports. \\
\hline Participants & Characteristics of the study or programme participants. \\
\hline Intervention & Description of the intervention. \\
\hline \multicolumn{1}{|l}{ Intervention mechanism } & Theory of change of intervention. \\
\hline Intervention structure & Detailed intervention components, delivery and facilitators. \\
\hline Intervention duration & Duration of intervention. \\
\hline Comparison & Description of any comparison groups. \\
\hline $\begin{array}{l}\text { Study outcomes and measures } \\
\text { Findings }\end{array}$ & List of outcomes and other measures assessed in study or programme evaluation. \\
\hline Recommendations & Main results of the study. \\
\hline
\end{tabular}


data within each of the subdomains outlined in table 2, working together to consolidate the findings through consensus. Additional content experts with knowledge of obstetric fistula in global contexts (AD, SO, JB, AK, JKB) will be available to provide guidance during this step. The text will describe how the findings of the scoping review relate to the review objective and respond to each of the specific research questions. We will then discuss our recommendations for future research and next steps.

\section{ETHICS AND DISSEMINATION}

No ethical review is required for a scoping review of the literature; thus, we have not sought ethics approval for our review.

The proposed scoping review will help in understanding and describing what is known and unknown about rehabilitation and social reintegration of women following genital fistula surgery. We know that as a consequence of fistula, women are often stigmatised and marginalised from their community, with high rates of depression that persist even after surgical repair. While access to surgical care has improved, there is less known about the approach to postsurgical social services, including reintegration and rehabilitation. While there is a call in the literature for the need of postsurgical reintegration services, there is a dearth of information describing best practices or detailing the specific requirements needed for a successful programme.

Results from this scoping review will be useful in developing social reintegration projects in areas where fistula projects are ongoing. The results of this review can be used to inform policy and serve as support for funding and development of reintegration programme. Furthermore, the results can highlight areas or future research to pursue and help in building protocols for postsurgical rehabilitation and reintegration programme throughout contexts where fistula is prevalent.

The study may be limited by including only articles published in English and French as study eligibility criteria and the lack of published articles on this topic. However, our scoping review will also include a systematic approach to reviewing the grey literature, in hopes of capturing all relevant information. Even in finding a deficit of published information, this scoping review may be a call for future published research in the area of social reintegration and rehabilitation of women following genital fistula surgery.

\section{Author affiliations}

${ }^{1}$ Department of Obstetrics, Gynecology and Reproductive Sciences, University of California, San Francisco, San Francisco, California, USA

${ }^{2}$ Department of Obstetrics and Gynecology, Urogynecology Division, Kaiser Permanente, Oakland, California, USA

${ }^{3}$ Department of Public Health, Gamal Abdel Nasser University, Conakry, Guinea ${ }^{4}$ ZSFG Library, University of California, San Francisco, San Francisco, California, USA ${ }^{5}$ Department of Obstetrics and Gynaecology, Mulago National Referral Hospital, Kampala, Uganda

${ }^{6}$ Department of Obstetrics and Gynaecology, Makerere University, Kampala, Uganda ${ }^{7}$ Department of Maternal and Child Health, Uganda Christian University, Mukono, Uganda
Acknowledgements Publication made possible in part by support from the UCSF Open Access Publishing Fund.

Contributors AME, JB, SO and JKB conceived the project. AME, CEP and JB-W drafted the protocol. AME, CEP, AD, JB-W, SO, JB, AK and JKB revised the protocol. All authors agreed upon the final protocol version.

Funding This work will be supported in part by the Eunice Kennedy Shriver National Institute of Child Health and Human Development grant number R0OHD086232.

Competing interests None declared.

Patient consent for publication Not required.

Provenance and peer review Not commissioned; externally peer reviewed.

Open access This is an open access article distributed in accordance with the Creative Commons Attribution Non Commercial (CC BY-NC 4.0) license, which permits others to distribute, remix, adapt, build upon this work non-commercially, and license their derivative works on different terms, provided the original work is properly cited, appropriate credit is given, any changes made indicated, and the use is non-commercial. See: http://creativecommons.org/licenses/by-nc/4.0/.

ORCID iD

Alison M El Ayadi http://orcid.org/0000-0003-2674-4887

\section{REFERENCES}

1 de Bernis L. Obstetric fistula: guiding principles for clinical management and programme development, a new who guideline. Int J Gynaecol Obstet 2007;99:S117-21.

2 Wall LL. Obstetric vesicovaginal fistula as an international publichealth problem. Lancet 2006;368:1201-9.

3 Hilton P. Vesico-vaginal fistulas in developing countries. Int $J$ Gynaecol Obstet 2003;82:285-95.

4 Hilton P, Ward A. Epidemiological and surgical aspects of urogenital fistulae: a review of 25 years' experience in Southeast Nigeria. Int Urogynecol J Pelvic Floor Dysfunct 1998;9:189-94.

5 Arrowsmith S, Hamlin EC, Wall LL. Obstructed labor injury complex: obstetric fistula formation and the multifaceted morbidity of maternal birth trauma in the developing world. Obstet Gynecol Surv 1996;51:568-74.

6 Wall LL, Arrowsmith SD, Briggs ND. Urinary Incontinence in the Developing World: The Obstetric Fistula. In: Abrams P, Cardozo L, Khoury S, et al, eds. Incontinence. Plymouth, UK: Health Publication Ltd, 2002.

7 Wall LL, Arrowsmith SD, Briggs ND, et al. The obstetric vesicovaginal fistula in the developing world. Obstet Gynecol Surv 2005;60:S3-51.

8 Zeleke BM, Ayele TA, Woldetsadik MA, et al. Depression among women with obstetric fistula, and pelvic organ prolapse in Northwest Ethiopia. BMC Psychiatry 2013;13:236.

9 Browning A, Fentahun W, Goh JTW. The impact of surgical treatment on the mental health of women with obstetric fistula. BJOG 2007;114:1439-41.

10 Goh JTW, Sloane KM, Krause HG, et al. Short communication: mental health screening in women with genital tract fistulae. BJOG 2005;112:1328-30.

11 Delamou A, Utz B, Delvaux T, et al. Pregnancy and childbirth after repair of obstetric fistula in sub-Saharan Africa: Scoping Review. Trop Med Int Health 2016;21:1348-65.

12 Kopp DM, Wilkinson J, Bengtson A, et al. Fertility outcomes following obstetric fistula repair: a prospective cohort study. Reprod Health 2017;14:159.

13 Delamou A, Delvaux T, El Ayadi AM, et al. Fistula recurrence, pregnancy, and childbirth following successful closure of female genital fistula in guinea: a longitudinal study. Lancet Glob Health 2017;5:e1152-60.

14 El Ayadi AM NH, Barageine J, Obore S, et al. Trajectories of Women's Physical and Psychosocial Recovery following Fistula Repair in Uganda. Tropical Medicine \& International Health. In Press.

15 Ahmed S, Holtz SA. Social and economic consequences of obstetric fistula: life changed forever? Int J Gynaecol Obstet 2007;99:S10-15.

16 Lombard L, de St. Jorre J, Geddes R, et al. Rehabilitation experiences after obstetric fistula repair: systematic review of qualitative studies. Tropical Medicine \& International Health 2015;20:554-68.

17 Ojengbede OA, Baba Y, Morhason-Bello IO, et al. Group psychological therapy in obstetric fistula care: a complementary recipe for the accompanying mental ill health morbidities? Afr $J$ Reprod Health 2014;18:155-9. 
18 Johnson KA, Turan JM, Hailemariam L, et al. The role of counseling for obstetric fistula patients: lessons learned from Eritrea. Patient Educ Couns 2010;80:262-5.

19 Arskey H, O'Malley L. Scoping studies: towards a methodological framework. International Journal of Social Research Methodology 2005;8:19-32.

20 Levac D, Colquhoun H, O'Brien KK. Scoping studies: advancing the methodology. Implement Sci 2010;5.

21 Tricco AC, Lillie E, Zarin W, et al. PRISMA extension for scoping reviews (PRISMA-ScR): checklist and explanation. Ann Intern Med 2018;169:467-73.
22 Health Sciences LIbrary System UoP. Systematic review workshop, 2018. Available: https://www.hsls.pitt.edu/systematicreview

23 McGowan J, Sampson M, Salzwedel DM, et al. PRESS Peer Review of Electronic Search Strategies: 2015 Guideline Statement. J Clin Epidemiol 2016;75:40-6.

24 The Joanna Briggs Institute. Joanna Briggs Institute reviewers' manual: 2015 edition/supplement. Adelaide: The Joanna Briggs Institute, 2015. 